47 Neuschwander-Tetri BA, Brunt EM, Wehmeier KR, Oliver D, Bacon BR. Improved nonalcoholic steatohepatitis after 48 weeks of treatment with the PPAR-gamma ligand rosiglitazone. Hepatology 2003;38:1008-17.

48 Laurin J, Lindor KD, Crippin JS, Gossard A et al. Ursodeoxycholic acid or clofibrate in the treatment of non-alcoholic-induced steatohepatitis: a pilot study. Hepatology 1996;23:1464-7.

49 Chalasani N, Aljadhey H, Kesterson J, Murray MD, Hall SD. Patients with elevated liver enzymes are not at higher risk for statin hepatotoxicity. Gastroenterology 2004;126: 1287-92.

50 Yoshiji H, Kuriyama S, Yoshii J, Ikenaka Y et al. Angiotensin-II type 1 receptor interaction is a major regulator for liver fibrosis development in rats. Hepatology 2001; 34:745-50.

51 Yokohama S, Yoneda M, Haneda M, Okamoto S et al. Therapeutic efficacy of an angiotensin II receptor antagonist in patients with nonalcoholic steatohepatitis. Hepatology 2004;40:1222-5.

52 Merat S, Malekzadeh R, Sohrabi MR, Sotoudeh $\mathrm{M}$ et al. Probucol in the treatment of non-alcoholic steatohepatitis: a double-blind randomized controlled study. J Hepatol 2003;38:414-8.

53 Abdelmalek MF, Angulo P, Jorgensen RA, Sylvestre PB, Lindor KD. Betaine, a promising new agent for patients with nonalcoholic steatohepatitis: results of a pilot study. Am J Gastroenterol 2001;96:2711-7.

54 Lavine JE. Vitamin E treatment of nonalcoholic steatohepatitis in children: a pilot study. J Pediatr 2000;136:734-8.

55 Harrison SA, Torgerson S, Hayashi P, Ward J, Schenker S. Vitamin E and vitamin C treatment improves fibrosis in patients with nonalcoholic steatohepatitis. Am J Gastroenterol 2003;98:2485-90.

56 Adams LA, Zein CO, Angulo P, Lindor KD. A pilot trial of pentoxifylline in nonalcoholic steatohepatitis. Am J Gastroenterol 2004;99:2365-8.

57 Yuan M, Konstantopoulos N, Lee J, Hansen $\mathrm{L}$ et al. Reversal of obesity- and diet-induced insulin resistance with salicylates or targeted disruption of IKKbeta. Science 2001;293: 1673-7.

58 Lindor KD, Kowdley KV, Heathcote EJ, Harrison ME et al. Ursodeoxycholic acid for treatment of nonalcoholic steatohepatitis: results of a randomized trial. Hepatology 2004;39:770-8.

59 Contos MJ, Cales W, Sterling RK, Luketic VA et al. Development of nonalcoholic fatty liver disease after orthotopic liver transplantation for cryptogenic cirrhosis. Liver Transpl 2001;7:363-73.

60 Ong J, Younossi ZM, Reddy V, Price LL et al. Cryptogenic cirrhosis and posttransplantation nonalcoholic fatty liver disease. Liver Transpl 2001;7:797-801.

\section{Autoimmune hepatitis}

Stefan Lueth MD, Registrar

Ansgar W Lohse MD, Professor of

Gastroenterology and Hepatology

Department of Medicine, University Medical Centre Hamburg-Eppendorf, Hamburg, Germany

\section{Clin Med 2006;6:25-8}

Autoimmune hepatitis (AIH) is a chronic inflammatory disorder of the liver associated with hypergammaglobulinaemia and the occurrence of autoantibodies. ${ }^{1,2}$ It was the first liver disease in which controlled trials proved both the efficacy of a therapeutic intervention (corticosteroids) and that timely diagnosis and adequate therapy can change a dismal prognosis into a normal life expectancy in the vast majority of patients. ${ }^{3}$ AIH describes a complex disease entity with a wide range of clinical presentations. one in 5,000-10,000. ${ }^{4}$ It can manifest at
The prevalence of AIH is estimated as any age between very early childhood and the 80 s and, like many other autoimmune diseases, is more common in women (3:1). AIH often presents subclinically ${ }^{5}$ leading to delayed diagnosis, which explains why about a quarter of patients already show cirrhosis at presentation. ${ }^{6}$ Up to one-third of patients may present with an acute disease, some even with fulminant hepatic failure. Delay in diagnosis and initiating treatment in such patients can be fatal.

\section{Diagnosis}

A diagnosis of AIH should be considered in any patient with elevated liver enzymes. The clinical picture only occasionally provides important clues to the diagnosis. The typical patient has subacute disease, presenting with lethargy and often jaundice without a risk factor for viral hepatitis. Other clinical symptoms may include arthralgia and slight, right upper quadrant pain.

With the possible exception of
Table 1. Criteria for the diagnosis of autoimmune hepatitis (AlH).

\section{Criteria}

$\begin{array}{ll}\text { Major } & \text { Hypergammaglobulinaemia (with a preferential increase of lgG) } \\ & \text { Demonstration of autoantibodies (ANA, SMA, SLA/LP, LKM) } \\ & \text { Absence of viral hepatitis } \\ & \text { Portal hepatitis (with lymphoplasmacellular infiltrates) on histology } \\ \text { Minor* } & \text { Personal or family history of other autoimmune disease } \\ & \text { History of a spontaneously fluctuating course } \\ & \text { Arthralgia } \\ & \text { Presence of HLA-DR3 or DR4 }\end{array}$

* additional features may give further support to the diagnosis.

ANA = antinuclear antibody; lgG = immunoglobulin G; LKM = liver-kidney microsomal antigen; SLA/LP = soluble liver antigen; SMA = smooth muscle antigen. 
anti-soluble liver antigen (SLA/LP) antibodies, there is no single test that proves or disproves the diagnosis of AIH. Diagnosis is therefore based on the combination of history, clinical examination and several laboratory and serological variables, the key features of which are described in Table 1, together with liver biopsy findings. The usually prompt response to immunosuppression proves the diagnosis.

\section{Screening tests}

As a first step, viral hepatitis B and C should be excluded by testing for hepatitis B surface antigen and antihepatitis $\mathrm{C}$ virus. Alcohol consumption, intake of hepatotoxic medication and hereditary liver diseases (Wilson disease, haemochromatosis, alpha-1 antitrypsin deficiency) should be excluded. The first hint to the presence of AIH is demonstration of elevated gammaglobulins on serum electrophoresis. This is probably the cheapest screening test for $\mathrm{AIH}$, but $5-10 \%$ of AIH patients do not display elevated gammaglobulins at initial diagnosis. Serum lipids should be tested as fatty liver disease is an important differential diagnosis.

Quantitative immunoglobulin (Ig) A, IgG and IgM levels are also cheap to test and are helpful diagnostic markers. In $\mathrm{AIH}$ there is typically selective elevation of IgG, with normal levels of IgA and IgM. Elevated IgA suggests a toxic cause, particularly alcohol, while elevated IgM is a feature of biliary disease, particularly primary biliary cirrhosis (PBC). Both $\mathrm{PBC}$ and primary sclerosing cholangitis (PSC) can be associated with AIH so-called overlap syndromes (see below).

\section{Autoantibodies}

At least $80 \%$ of $\mathrm{AIH}$ patients have pathological titres of at least one autoantibody. The most common are antinuclear antibodies (ANA) and antibodies to smooth muscle antigen (SMA); both are present in $40-50 \%$ of cases, sometimes in combination. Antibodies to SLA/LP are present in about $20 \%$, in half of whom they are the only autoantibody demonstrable. Antibodies to liver-kidney microsomal antigen (LKM) are uncommon (1-3\%) but delineate a separate disease subgroup often called AIH type 2; this is more prevalent in childhood where it may be a severe disease.

There are two methods for testing autoantibodies:

- ANA, SMA and antibodies to LKM are best tested using immunofluorescence on tissue

\section{Key Points}

\begin{abstract}
Autoimmune hepatitis $(\mathrm{AlH})$ can occur in all age groups from earliest childhood until the ninth decade

\section{AlH affects women more commonly than men (3:1)}

Clinical presentation may range from acute hepatitis to fulminant liver failure but can also be asymptomatic

\section{Diagnosis is based on a combination of elevated immunoglobulin (gammaglobulin) levels, autoantibodies and histological proof of hepatitis in the absence of active viral markers}

\section{Response to immunosuppression is universal and prompt}

Steroids are the drug of choice for remission induction and azathioprine for maintenance of remission

Treatment should be maintained for a minimum of three years, but is often required lifelong

KEY WORDS: autoantibodies, cirrhosis, gammaglobulin, immunosuppression, transaminases sections. This will also detect antimitochondrial antibodies as the characteristic test for PBC.

- For SLA/LP antibodies, which do not show up on immunofluorescence and are therefore missed by routine testing, specific immunoassays such as ELISA and/or immunoblot should be used.

ANA, SMA and LKM can also be found in some patients with other liver disease and non-hepatic autoimmune disease, but SLA/LP antibodies are highly specific for AIH and thus diagnostic in patients in whom they can be demonstrated. This also seems to be true for antibodies to double-stranded DNA (detectable in 15\%), for which the only differential diagnosis is systemic lupus erythematosus (SLE).

\section{Histology}

The diagnosis of AIH should also be based on a liver biopsy. This is required both to demonstrate the presence of a lymphocellular infiltrate and also to exclude important differential diagnoses such as drug-induced liver disease or non-alcoholic steatohepatitis. About a quarter of patients with $\mathrm{AIH}$ already have cirrhosis at the time of diagnosis. ${ }^{6}$ Cirrhosis is frequently macronodular; it may often be missed because of a sampling error in which biopsy of a large regenerative nodule misses the fibrous septa. Some authors therefore favour laparoscopy at initial diagnosis; this can now be performed with minimal invasiveness due to the availability of mini-instruments.

\section{Treatment}

Immunosuppression dramatically improves the outcome in AIH. With individualised immunosuppression, patients now look forward to normal life expectancy with a good quality of life. Prompt response to sufficient doses of corticosteroids is a universal feature of $\mathrm{AIH}$, making it the most reliable final diagnostic tool. Non-response to immunosuppression suggests another 
diagnosis, provided that the patient is compliant and the dose adequate.

In most patients, treatment monitoring can be based solely on laboratory investigations (eg aminotransferases and gammaglobulins). Liver biopsy on follow-up is required only in special situations.

\section{Remission}

\section{Induction}

Different experts recommend different doses. We strongly recommend a starting dose of $1 \mathrm{mg} / \mathrm{kg}$ prednisolone for all patients except those with mild disease. Severe icteric disease may cause malabsorption of corticosteroids so intravenous (iv) administration is best. Transaminase levels usually fall within a few days and the prednisolone dose can soon be tapered. A typical treatment schedule for a patient with a body weight of $75 \mathrm{~kg}$ is given in Table 2 .

The dose can be tapered more quickly in patients who have a prompt response and in milder disease. However, it should not be reduced too quickly because rapid tapering often results in early relapse, thus requiring higher overall steroid doses. Administration of vitamin D and calcium prevents development of osteoporosis.

\begin{tabular}{|c|c|}
\hline Week & $\begin{array}{l}\text { Corticosteroids } \\
\text { (mg/day) }\end{array}$ \\
\hline 1 & 75 \\
\hline 2 & 60 \\
\hline 3 & 50 \\
\hline 4 & 40 \\
\hline 5 & 30 \\
\hline 6 & 25 \\
\hline 7 & 20 \\
\hline $8 \& 9$ & 15 \\
\hline $10 \& 11$ & 12.5 \\
\hline $12 \ldots$ & 10 \\
\hline
\end{tabular}

\section{Maintenance}

Steroids are the drug of choice for the induction of remission but azathioprine (AZT) is the drug of choice for maintenance of remission. Its effect takes several weeks to develop fully, so AZT should be started as soon as the diagnosis of $\mathrm{AIH}$ has been established; it may then allow the dose of corticosteroids to be reduced. The usual dose of azathioprine is $1 \mathrm{mg} / \mathrm{kg}$ (considerably lower than in inflammatory bowel disease) and tolerability is very good in most patients.

If the diagnosis has not been confirmed at the time of initiating treatment, the response to steroids should be evaluated before adding AZT. In icteric patients the pharmacodynamics of AZT and its metabolites may be considerably altered, increasing the danger of toxicity. In these patients a fall of bilirubin below 150 microgram/ml should normally first be achieved before adding AZT, using a lower starting dose and gradually increasing to the desired dose.

An idiosyncratic reaction may occur in about $3 \%$ of patients causing abdominal pain, nausea and fever. In addition, bone marrow toxicity is possible so regular blood counts are required, initially every 1-2 weeks then slowly decreasing to once every three months. The superiority of AZT over corticosteroids in maintaining remission has been convincingly demonstrated by the King's College group. ${ }^{7}$

Combination therapy should be given for the first 12 months, then corticosteroids should be tapered slowly for all patients in biochemical remission (normal transaminases and normal IgG). Some groups recommend increasing the dose of AZT at this stage up to $2 \mathrm{mg} / \mathrm{kg}$ to avoid relapse on steroid withdrawal, others (including our group) prefer to continue low-dose steroids $(5 \mathrm{mg}$ ) and low-dose azathioprine $(1 \mathrm{mg} / \mathrm{kg})$ because of worries about oncogenic effects of long-term high-dose AZT. Transaminases and IgG should be monitored closely each time immunosuppression is significantly decreased as relapse upon dose reduction is not uncommon. Relapse may often be preceded by an increase in IgG well before the transaminases increase; if this occurs, the original regimen should be reinstituted immediately. When transaminases rise, steroid dosage should be increased briefly. The exact dose and duration of the increase depend on when a relapse is detected: if the intervention is early in the relapse, an increase for only a limited time is required (eg $20 \mathrm{mg}$ for 1 week, $15 \mathrm{mg}$ for 1 week, $10 \mathrm{mg}$ for 2 weeks), returning to the original maintenance dose thereafter.

\section{Treatment withdrawal}

Most patients will require long-term (mostly lifelong) immunosuppression, many being maintained on 50-100 mg AZT daily. AZT should be continued for a minimum of three years in all patients before complete treatment withdrawal is considered, otherwise relapse rates are high (>80\% within 18 months). ${ }^{8}$ Treatment withdrawal should be considered in patients who have been relapse-free for three years in whom liver biopsy has demonstrated absence of significant inflammation. The strongest predictor of relapse after treatment withdrawal is the presence of histological signs of continued inflammation. ${ }^{9}$

Compliance may become a problem in some long-term patients. It may occasionally be justified to attempt treatment withdrawal despite negative predictors for stable remission, as only proof of relapse will convince these patients to take a potentially toxic drug for many years.

Patients must be closely observed for the first 1-2 years after treatment withdrawal in order to detect relapse which may be delayed by many months. It is important to emphasise the risk of late relapse to patients and their caregivers so that they are not misled by a stable situation early after treatment withdrawal.

\section{Difficult-to-treat patients}

\section{Fulminant autoimmune hepatitis}

A few patients, more commonly children and young adults, present with fulminant hepatic failure. Unfortunately, immunosuppression may be too late for some of them. The standard criteria for emergency liver transplantation should 
be used for these patients, despite the presence of an underlying condition which, in principle, is responsive to medical treatment. Some patients rapidly deteriorate on steroids in this situation and any delay in transplantation will increase the risk of potentially fatal infection. Patients without encephalopathy, however, should undergo an intensive trial of immunosuppression with up to $250 \mathrm{mg}$ prednisolone iv for the first few days.

More intensive immunosuppression, including AZT, is recommended following liver transplantation as relapse of $\mathrm{AIH}$ is observed in up to a fifth of organ recipients. ${ }^{10}$

\section{Patients intolerant of azathioprine}

About $3 \%$ of patients are intolerant to AZT and a similar proportion will develop side effects later (myelosuppression, hepatotoxicity, pancreatitis) requiring either dose reduction or drug withdrawal. Some of these patients can be managed with steroid monotherapy, but most will require another secondline drug. Mycophenolate mofetil (MMF) has emerged as the standard second-choice drug, primarily because of its low toxicity. There are only limited data but the overall experience is that about two-thirds of patients fare well on MMF 1-2 g/day.

In the one-third who fail to benefit from MMF the choice of drug is more difficult. Our preference (in descending order) is cyclophosphamide, methotrexate or cyclosporin. Cyclophosphamide can be given orally (100 mg/day initially) or as a bolus infusion as in SLE. These patients should be treated in specialised centres as side effects of the drug need to be closely weighed against the dangers of progressive disease.

\section{Poor responders to standard immunosuppression}

Few patients fail to reach remission using the schedule of high-dose steroids in the initiation of treatment. The best choice of management in some of the non-responders may be higher than usual maintenance doses (10-20 mg/day prednisolone and $\leq 2 \mathrm{mg} / \mathrm{kg} /$ day AZT).

Only exceptional patients will continue to show high inflammatory activity despite such a treatment schedule. It is important to re-examine the diagnosis and to exclude drug-induced hepatotoxicity; a second opinion, both clinically and on the histology, may also be helpful. Various experimental protocols have been used; our personal preference is again cyclophosphamide. In order to give specialist centres the chance to evaluate novel therapeutic approaches in such patients, they should be referred to experts with a special interest in AIH.

Discussion of these exceptional cases should not distract from the fact that $\mathrm{AIH}$ is a condition that can be managed extremely successfully by medical therapy which, however, needs to be individually tailored. With such careful management, both survival and quality of life are excellent.

\section{Overlap syndromes}

Some $10-20 \%$ of patients will have overlapping features with either PSC $^{11}$ or (more commonly) PBC. ${ }^{12}$ Management should be primarily directed by the $\mathrm{AIH}$ component of their disease. This is the part of the syndrome which drives more rapid liver damage and progression to cirrhosis and which can be treated effectively. In overlap patients it appears sensible to add ursodeoxycholic acid to the treatment schedule, even though the evidence for this approach is weak. Patients with AIH/PSC overlap seem to run a less favourable course than those with $\mathrm{AIH}$ only, while $\mathrm{AIH} / \mathrm{PBC}$ overlap patients seem to have a similarly good prognosis to that of AIH alone.

\section{References}

1 Mackay IR, Weiden S, Ungar B. Treatment of active chronic hepatitis and lupoid hepatitis with 6-mercaptopurine and azathioprine. Lancet 1964;42:899-902.

2 O'Brien EN, Goble AJ, Mackay IR. Plasmatransaminase activity as an index of the effectiveness of cortisone in chronic hepatitis. Lancet 1958;i:1245-9.

3 Krawitt EL. Autoimmune hepatitis. Review. N Engl J Med1996;334:897-903.

4 Boberg KM, Aadland E, Jahnsen J, Raknerud $\mathrm{N}$ et al. Incidence and prevalence of primary biliary cirrhosis, primary sclerosing cholangitis, and autoimmune hepatitis in a Norwegian population. Scand J Gastroenterol 1998;33:99-103.

5 Kogan J, Safadi R, Ashur Y, Shouval D, Ilan Y. Prognosis of symptomatic versus asymptomatic hepatitis: a study of 68 patients. J Clin Gastroenterol 2002;35:75-81.

6 Roberts SK, Therneau TM, Czaja AJ. Prognosis of histological cirrhosis in type 1 autoimmune hepatitis. Gastroenterology 1996;110:848-57.

7 King's College group.

8 Kanzler S, Löhr H, Gerken G, Galle PR, Lohse AW. Long-term management and prognosis of autoimmune hepatitis (AIH): a single center experience. $Z$ Gastroenterol 2001;39:339-41,344-8.

9 Kanzler S, Gerken G, Löhr H, Galle PR et al. Duration of immunosuppressive therapy in autoimmune hepatitis. J Hepatol 2001;34: 354-5.

10 Neuberger J, Portmann B, Calne R, Williams R. Recurrence of autoimmune chronic active hepatitis following orthotopic liver grafting. Transplantation 1984; 37:363-5.

11 Gohlke F, Lohse AW, Dienes HP, Lohr H et al. Evidence for an overlap syndrome of autoimmune hepatitis and primary sclerosing cholangitis. J Hepatol 1996;24: 699-705.

12 Lohse AW, zum Buschenfelde KH, Franz B, Kanzler $\mathrm{S}$ et al. Characterization of the overlap syndrome of primary biliary cirrhosis (PBC) and autoimmune hepatitis: evidence for it being a hepatitic form of PBC in genetically susceptible individuals. Hepatology 1999;29:1078-84. 